\title{
Analytical Model for Explosive-Driven Ultracompact Shock-Wave Ferromagnetic Generators
}

\author{
E.F. Thlantsev ${ }^{a}$, S.I. Shkuratov ${ }^{b, *}$, J. Baird ${ }^{b}$ And L.L. Altgilbers ${ }^{c}$ \\ ${ }^{a}$ Pulsed Power LLC, Lubbock, TX 79416, USA \\ ${ }^{b}$ Loki, Incorporated, Rolla, MO 64509, USA \\ ${ }^{c}$ U.S. Army Space and Missile Defense Command/Army Strategic Command \\ Huntsville, AL 35807, USA
}

\begin{abstract}
An analytical model of the operation of recently invented ultracompact explosive-driven shock-wave ferromagnetic generators that utilize longitudinal (shock wave propagates along the magnetization vector) and transverse (shock wave propagates across the magnetization vector) shock wave demagnetization of $\mathrm{Nd}_{2} \mathrm{Fe}_{14} \mathrm{~B}$ hard ferromagnets, and an analytical technique for calculation of amplitudes of the current pulse produced by the generators were developed. The model utilizes the magnitude of the energy product, $(B H)_{\max }$, and the magnetostatic energy of the hard ferromagnetic element of the ferromagnetic generator as parameters for determining ferromagnetic generator pulse generation, and it does not consider the magnitude of remnant magnetization, $B_{\mathrm{r}}$, of the ferromagnetic material. The developed technique for calculation of the current pulse amplitude produced by the ferromagnetic generator provides comprehensive and accurate results. Amplitudes of calculated ferromagnetic generator output currents are in good agreement with experimentally obtained data.
\end{abstract}

PACS numbers: $62.50 .+\mathrm{p}, 75.50 . \mathrm{Ww}, 43.40 . \mathrm{Jc}$

\section{Introduction}

Generation of primary electrical power by compact autonomous explosively driven sources is critical to the success of many modern scientific and engineering projects. Two new types of miniature generators of primary power have recently been developed by Shkuratov et al. [1-11]. These generators are powered by the chemical energy of high explosives. Modern energetic nanocomposites [12] can make the dimensions of these electrical devices even smaller. The first type of primary power generators is based on longitudinal shock depolarization of high-energy ferroelectric materials [1-3]. Theoretical modelling of the operation of generators of this type is a very challenging task because the ferroelectric module is always connected to the load, but the electrical parameters of the ferroelectric material are practically unpredictable because it is subjected to shock wave compression [4].

The original basic idea of recently invented primary power sources of the second type [5-11] was to utilize magnetostatic energy stored exclusively in modern hard ferromagnetic materials possessing high energy products, $(B H)_{\max }$, instead of soft ferromagnetic materials possessing very high residual magnetization, $B_{\mathrm{r}}$, but extremely low coercive force, $H$ (and correspondingly low energy product). These primary electrical sources are based on physical effects of longitudinal (shock wave propa-

* corresponding author; e-mail: shkuratov@lokiconsult.com gates along the magnetization vector) [5] and transverse (shock wave propagates across the magnetization vector) [6-11] demagnetization of hard ferromagnetic energy-carrying elements by shock waves generated by high explosives. It was experimentally demonstrated that miniature explosively driven shock wave ferromagnetic generators (FMGs) containing $\mathrm{Nd}_{2} \mathrm{Fe}_{14} \mathrm{~B}$ hard ferromagnetic elements possessing high $(B H)_{\max }$ generate output currents with amplitudes up to $5 \mathrm{kA}[6,7,9]$ and output voltages with amplitudes up to $50 \mathrm{kV}[7,10,11]$.

The shock-wave FMGs [5-11] contain $\mathrm{Nd}_{2} \mathrm{Fe}_{14} \mathrm{~B}$ hard ferromagnetic energy elements, high explosive charges and pulse-generating coils wound on the $\mathrm{Nd}_{2} \mathrm{Fe}_{14} \mathrm{~B}$ ferromagnets. Detailed description of the designs of longitudinal shock-wave FMGs can be found in [5] and transverse shock-wave FMGs in [6-11]. Operation of the FMG is as follows. After detonation of the FMG explosive charge, the $\mathrm{Nd}_{2} \mathrm{Fe}_{14} \mathrm{~B}$ hard ferromagnetic energy-carrying element is subjected to longitudinal or transverse shock-wave compression. The shock compression demagnetizes the $\mathrm{Nd}_{2} \mathrm{Fe}_{14} \mathrm{~B}$ hard ferromagnetic element, which generates a pulsed electromotive force (EMF) at the output terminals of the FMG pulse-generating coil. Thus, the initial magnetostatic energy of $\mathrm{Nd}_{2} \mathrm{Fe}_{14} \mathrm{~B}$ hard ferromagnet is transformed into pulsed power.

In this paper we present an analytical model that describes the operation of shock wave FMGs, and an analytical technique for calculating the output current pulse amplitudes produced by these generators. 


\section{FMG analytical model and technique for calculation of FMG current}

The initial electromagnetic energy of the FMG [5-11], $W_{\mathrm{FMG}}$, is equal to the initial magnetostatic energy of the $\mathrm{Nd}_{2} \mathrm{Fe}_{14} \mathrm{~B}$ hard ferromagnetic element, $W_{\mathrm{NdFeB}}$ :

$$
W_{\mathrm{FMG}}=W_{\mathrm{NdFeB}} \text {, }
$$

where $W_{\mathrm{NdFeB}}$ is the magnetic energy transformed into the energy of a current pulse. Assuming that the ohmic losses in the FMG-load circuit are negligible, the energy in the system during the generation process, $W_{\text {current }}$, is

$$
W_{\text {current }}=\left[L_{\text {total }}\left(I_{\max }\right)^{2}\right] / 2,
$$

where $I_{\max }$ is the amplitude of the current pulse. The total inductance of the system, $L_{\text {total }}$, is the sum of the inductances of the FMG and a load

$$
L_{\text {total }}=L_{\mathrm{FMG}}+L_{\text {Load }} \text {. }
$$

Taking into account that the FMG has an efficiency coefficient, $\eta_{\mathrm{FMG}}$, the final equation for initial energy in the FMG-load system can be expressed as follows:

$$
\begin{aligned}
& W_{\text {current }}= \\
& \eta_{\mathrm{FMG}} W_{\mathrm{NdFeB}}=\left[\left(L_{\mathrm{FMG}}+L_{\mathrm{Load}}\right)\left(I_{\mathrm{max}}\right)^{2}\right] / 2 .
\end{aligned}
$$

Equation (5) gives the amplitude of the current

$$
\begin{aligned}
& I_{\max }(t)=\eta_{\text {currentFMG }} \\
& \quad \times\left[\left(2 W_{\mathrm{NdFeB}}\right) /\left(L_{\mathrm{FMG}}+L_{\mathrm{Load}}\right)\right]^{1 / 2},
\end{aligned}
$$

where $\eta_{\text {currentFMG }}=\left(\eta_{\mathrm{FMG}}\right)^{1 / 2}$ is the coefficient of FMG efficiency for the conversion of $W_{\mathrm{NdFeB}}$ into a current pulse. $W_{\mathrm{NdFeB}}$ can be estimated by using

$$
W_{\mathrm{NdFeB}}=V(B H)_{\max } / 2,
$$

where $V$ is the volume of the $\mathrm{Nd}_{2} \mathrm{Fe}_{14} \mathrm{~B}$ FMG hard ferromagnetic element, and $(B H)_{\max }$ is the maximum energy product of the hard ferromagnetic material. Hence, from Eqs. (5) and (6), we can find the expression for $\eta_{\text {currentFMG: }}$

$$
\begin{aligned}
& \eta_{\text {currentFMG }}=I_{\max }(t) / \\
& {\left[\left(V(B H)_{\max }\right) /\left(L_{\mathrm{FMG}}+L_{\mathrm{Load}}\right)\right]^{1 / 2} .}
\end{aligned}
$$

We obtained Eqs. (5) and (7) as a direct result of the application of the energy conservation law to the FMG-load autonomous system. Moreover, we did not make any assumptions about the physical mechanism of shock-wave demagnetization of the ferromagnet. Thus, Eqs. (5) and (7) can be used for operational analysis of both longitudinal [5] and transverse [6-11] shock-wave FMGs.

Let us consider the current generation process based on the magnetic flux conservation law. The initial magnetic flux (before explosive operation) in the FMG-load system, $\Phi_{\mathrm{FMG}-\text { load }}$, is the magnetic flux that is coupled by the FMG pulse-generating coil wound on the $\mathrm{Nd}_{2} \mathrm{Fe}_{14} \mathrm{~B}$ hard ferromagnet $[5-11]$ :

$$
\Phi_{\text {FMG-load }}=\sum_{n=1}^{N} \Phi_{n},
$$

where $\Phi_{n}$ is the magnetic flux coupled by the $n$-th turn, and $N$ is the number of turns in the FMG coil. The value of $\Phi_{n}$ depends on the magnetic parameters and the shape of the ferromagnet, and on the disposition of the $n$-th turn on the ferromagnet [6]. Thus, $\Phi_{n}$ can be calculated. The methodology for calculating the magnetic flux coupled by the FMG pulse-generating coils was developed and described in Ref. [7].

During explosive operation, the $\mathrm{Nd}_{2} \mathrm{Fe}_{14} \mathrm{~B}$ element is subjected to shock wave compression and shock demagnetization [5-11]. The magnetic flux stored in the ferromagnet is transformed into the magnetic flux of the current, $\Phi_{\text {current }}(t)$, flowing through the FMG-load circuit

$$
\begin{gathered}
\Phi_{\text {FMG-load }}=\sum_{n=1}^{N} \Phi_{n}=\Phi_{\text {current }}(t)_{\max } \\
=\left(L_{\mathrm{FMG}}+L_{\text {Load }}\right) I_{\max }(t) .
\end{gathered}
$$

It follows from the flux conservation law that $\Phi_{\text {FMG-load }}$ must be conserved in the FMG-load system (with the assumption that losses are negligible). Thus, for $I_{\max }(t)$ in the FMG-load system we obtained a system of two independent equations. The first equation is Eq. (5). The second equation can be obtained from Eq. (9):

$$
I_{\max }(t)=\left(\sum_{n=1}^{N} \Phi_{n}\right) /\left(L_{\mathrm{FMG}}+L_{\mathrm{Load}}\right) .
$$

This system [Eqs. (5) and (10)] may be solved by the method of successive iterative calculations. The general method is as follows:

(1) Assuming that $L_{\mathrm{FMG}}=0$, Eq. (5) gives the first approximation for the current value, $I_{\max }^{1 \text { st approx }}$.

(2) The substitution of $I_{\max }^{1 \text { st approx }}$ in Eq. (10) gives the first approximation for the initial value in magnetic flux of the system,

$$
\Phi_{\mathrm{FMG}-\mathrm{load}}=\sum \Phi_{n}^{1 \text { st approx }} .
$$

(3) The $\Phi_{n}\left\{\Phi_{1}, \Phi_{2}, \ldots, \Phi_{n}\right\}$ matrix is used to find the number, $N$, and the dispositions for $N$ turns of the FMG coil that should be wound on the ferromagnet for a given value of $\Phi_{\mathrm{FMG}}$-load $(\mathrm{Eq} .(11))$. A detailed description of the technique used to calculate the magnetic flux coupled by an FMG coil can be found in [7]. Thus, the design of the FMG current source (number of turns, $N$, and dispositions of the turns) is calculated to the first approximation.

(4) This design has an exact value for FMG inductance, $L_{\mathrm{FMG}}^{1 \text { st iteration }}$. If one makes the assumption that $L_{\mathrm{FMG}}=L_{\mathrm{FMG}}^{1 \mathrm{st}}$ iteration, Eq. (5) gives the second approx-

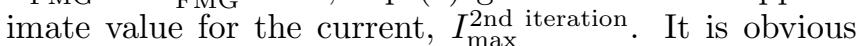
that $I_{\max }^{2 n d}$ iteration $<I_{\max }^{1 \mathrm{st}}$ iteration.

(5) The substitution of $I_{\max }^{2 n d}$ approx in Eq. (10) gives the second approximation for the initial magnetic flux in the FMG-load system, and so on. In practice, four iterations are enough to get $I_{\max }^{5 \text { th }}$ iteration $\approx I_{\max }^{4 \text { th iteration }}$.

To verify the technique developed in this paper for calculation of current pulse amplitudes produced by FMGs, we performed analysis of experimental data obtained in 
papers [5-9]. The amplitudes of output current pulses that were calculated with the developed technique are in very good agreement with those obtained experimentally. Based on these results, we conclude that the developed technique provides highly accurate calculation of amplitudes of output currents produced by high-current FMGs.

Based on the experimental data obtained in [5-9] and Eq. (7) we determined FMG coefficients for current and energy efficiency. For longitudinal FMG they are $\eta_{\text {currentLFMG }}=0.314 \pm 0.025$ and $\eta_{\text {energyLFMG }}=$ $0.099 \pm 0.06$. For transverse FMG they are $\eta_{\text {currentTFMG }}=0.392 \pm 0.015$ and $\eta_{\text {energyTFMG }}=$ $0.154 \pm 0.043$. The difference in the energy efficiency for longitudinal and transverse FMGs is probably related to the difference in the kinetics of the $\mathrm{Nd}_{2} \mathrm{Fe}_{14} \mathrm{~B}$ shock demagnetization processes, because the characteristic time for longitudinal shock demagnetization is longer [8] than the characteristic time for shock transverse demagnetization.

\section{References}

[1] S.I. Shkuratov, E.F. Talantsev, L. Menon, H. Temkin, J. Baird, L.L. Altgilbers, Rev. Sci. Instrum. 75, 2766 (2004).

[2] S.I. Shkuratov, E.F. Talantsev, J. Baird, M.F. Rose, Z. Shotts, L.L. Altgilbers, A.H. Stults, Rev. Sci. Instrum. 77, 043904 (2006).
[3] S.I. Shkuratov, E.F. Talantsev, J. Baird, A.V. Ponomarev, L.L. Altgilbers, A.H. Stults, IEEE Trans. Plasma Sci. 36, 44 (2008).

[4] Y. Tkach, S.I. Shkuratov, E.F. Talantsev, M. Kristiansen, J. Dickens, L.L. Altgilbers, P.T. Tracy, IEEE Trans. Plasma Sci. 30, 1665 (2002).

[5] S.I. Shkuratov, E.F. Talantsev, J.C. Dickens, M. Kristiansen, IEEE Trans. Plasma Sci. 30, 1681 (2002).

[6] S.I. Shkuratov, E.F. Talantsev, J.C. Dickens, M. Kristiansen, J. Appl. Phys. 92, 159 (2002).

[7] S.I. Shkuratov, E.F. Talantsev, J.C. Dickens, M. Kristiansen, J. Appl. Phys. 93, 4529 (2003).

[8] E.F. Talantsev, S.I. Shkuratov, J.C. Dickens, M. Kristiansen, Mod. Phys. Lett. B 16, 545 (2002).

[9] E.F. Talantsev, S.I. Shkuratov, J.C. Dickens, M. Kristiansen, Rev. Sci. Instrum. 74, 225 (2003).

[10] S.I. Shkuratov, E.F. Talantsev, J. Baird, L.L. Altgilbers, A.H. Stults, Rev. Sci. Instrum. 77, 066107 (2006).

[11] S.I. Shkuratov, E.F. Talantsev, J. Baird, L.L. Altgilbers, A.H. Stults, IEEE Trans. Plasma Sci. $\mathbf{3 4}$ 1866 (2006).

[12] L. Menon, S. Patibandla, K.B. Ram, S.I. Shkuratov, M. Holtz, H. Temkin, Appl. Phys. Lett. 84, 4735 (2004). 